\title{
Effect of preoperative low serum albumin on postoperative complications and early mortality in patients undergoing transcatheter aortic valve replacement
}

\author{
Asmae Gassa ${ }^{1 *}$, Jan H. Borghardt ${ }^{2 *}$, Johanna Maier ${ }^{1}$, Kathrin Kuhr ${ }^{3}$, Maximilian Michel ${ }^{4}$, Svenja Ney ${ }^{5}$, \\ Kaveh Eghbalzadeh ${ }^{1}$, Anton Sabashnikov ${ }^{1}$, Tanja Rudolph ${ }^{5}$, Stephan Baldus ${ }^{5}$, Navid Mader ${ }^{1 \#,}$ \\ Thorsten Wahlers ${ }^{1 \#}$
}

${ }^{1}$ Department of Cardiothoracic Surgery, Heartcenter, ${ }^{2}$ School of Medicine, ${ }^{3}$ Institute of Medical Statistics and Computational Biology, Medical Faculty, ${ }^{4}$ Institute of Zoology, ${ }^{5}$ Department of Internal Medicine III, Heartcenter, University of Cologne, Cologne, Germany

Contributions: (I) Conception and design: A Gassa, JH Borghardt, N Mader; (II) Administrative support: T Wahlers, S Baldus, T Rudolph; (III) Provision of study materials or patients: A Gassa, JH Borghardt, S Ney, K Eghbalzadeh, J Maier; (IV) Collection and assembly of data: A Gassa, JH Borghardt; (V) Data analysis and interpretation: A Gassa, JH Borghardt, K Kuhr, M Michel, A Sabashnikov; (VI) Manuscript writing: All authors; (VII) Final approval of manuscript: All authors.

*These authors are co-first authors.

\#These authors contributed equally to this work.

Correspondence to: Dr. med. Asmae Gassa. Department of Cardiothoracic Surgery, Heartcenter, University of Cologne, Kerpener Strasse 62, 50931

Cologne, Germany. Email: asmae.gassa@uk-koeln.de.

Background: Patients undergoing transcatheter aortic valve replacement (TAVR) are mostly elderly patients with substantial comorbidities. Established risk scores are not validated for TAVR and collectives with elderly patients making periprocedural risk stratification difficult. Serum albumin is known to be an indicator for malnutrition and frailty and is simple to measure, independent of physician's bias. Using serum albumin as a preoperative marker for postoperative complications might help estimating morbidity and mortality of these patients.

Methods: A total of 457 patients with severe symptomatic aortic stenosis undergoing TAVR at our institution in a period from January 2014 to December 2015 were included in this retrospective study. Baseline characteristics as well as preoperative laboratory parameters were registered. Postoperative morbidity and 30-day mortality were analyzed as primary end points. Enrolled patients with preoperative low serum albumin $(<3.5 \mathrm{~g} / \mathrm{dL})$ were compared with those revealing normal serum albumin $(\geq 3.5 \mathrm{~g} / \mathrm{dL})$.

Results: Among 457 patients, 51 (11\%) presented pre-procedural low serum albumin and 406 (89\%) had normal serum albumin. Patients' mean age was $81 \pm 6$ years and $50 \%$ of them were male. Postoperative complications such as requirement of blood transfusions (63\% versus $33 \%, \mathrm{P}<0.001)$, infection $(53 \%$ versus $24 \%, \mathrm{P}<0.001$ ), acute kidney injury (41\% versus $19 \%, \mathrm{P}=0.001)$ and 30 -day mortality (10\% versus $3 \%$, $\mathrm{P}=0.045$ ) showed significant differences between preoperative low and normal albumin groups.

Conclusions: Preoperative low serum albumin might be an indicator for higher morbidity and mortality in patients undergoing TAVR.

Keywords: Transcatheter aortic valve replacement (TAVR); hypoalbuminemia; postoperative complications

Submitted Jul 15, 2018. Accepted for publication Nov 06, 2018.

doi: $10.21037 /$ jtd.2018.11.30

View this article at: http://dx.doi.org/10.21037/jtd.2018.11.30 


\section{Introduction}

In recent years, transcatheter aortic valve replacement (TAVR) has become crucial to interventional therapy for patients with severe symptomatic aortic stenosis. Most of the patients undergoing TAVR are in poor medical conditions and of advanced age (1). Surgical treatment by means of conventional aortic valve replacement in these cases is associated with a higher risk of mortality (2-4). At the same time conservative standard treatment options are limited. $50 \%$ of patients with symptomatic aortic stenosis who are being treated conservatively die within 2 years $(5,6)$. This stands in contrast to patients undergoing TAVR where 1 -year mortality is around $17 \%(7)$.

However, a significant problem in using TAVR is the lack of an established risk evaluation system in order to identify patients who could profit from TAVR at an acceptable risk to the patient. This stands in contrast to more conventional surgeries such as intubation, thoracotomy or a heart-lung machine which all have well established risk score systems, for example the logistic European System for Cardiac Operative Risk Evaluation (log Euroscore) (8) or the Society of Thoracic Surgeons' Risk Score (STS) (9). Further complicating matters are that these scores were designed for a patient population with a lower mean age [e.g., $62.5 \pm 10.7$ years in the Euroscore database as opposed to 81.5 for TAVR (PARTNER II Trial)].

Serum albumin is synthesized in the liver and is the main plasma protein regulating colloid-osmotic pressure. Hypoalbuminemia [defined as serum albumin $<3.5 \mathrm{~g} / \mathrm{dL}$ (10)] is associated with frailty and malnutrition and could potentially be used as a biomarker. It is easy to quantify, part of most standard preoperative laboratory diagnostics and importantly, albumin levels are not vulnerable to short-term variation. Hypoalbuminemia has been found to be predictive for acute kidney injury (AKI) (11) and is further connected to higher infection rates and mortality $(12,13)$. We therefore carried out a retrospective study in order to establish the prognostic value of serum albumin in predicting postoperative complications such as AKI, blood transfusion rates, infection, elongation of hospitalization and early mortality in patients who undergo TAVR at a high-volume single center in Germany.

\section{Methods}

\section{Patients and data acquisition}

Records for 457 patients who underwent the TAVR procedure in a period from January 2014 to December
2015 in the Department of Cardiothoracic Surgery and the Department of Internal Medicine III at the University of Cologne, Germany were collected. All data including preoperative baseline characteristics, laboratory parameters, postoperative complications, length of hospital stay (LOS) and mortality were extracted from clinical documentation such as general medical reports, documented anamnesis at the time of the procedure, surgery reports, intensive or intermediate care unit reports and medical reports after the procedure. Institutional Review Board at the University of Cologne waived consent. Kidney Disease Improving Global Outcomes (KDIG) standards for AKI were used to interpret pre- and post-procedural creatinine values in order to complete data on post-interventional AKI. The postoperative 30-day evaluation for patients who underwent TAVR was assessed for 30-day mortality rates. The log Euroscore and STS risk score were calculated for every patient respectively using collected data.

Serum albumin analysis is part of the standard preprocedural blood diagnostics at our institution and was performed using the bromocresol green method (14). To analyze the impact of pre-procedural serum albumin, patients were categorized into a low $(<3.5 \mathrm{~g} / \mathrm{L})$ and normal serum albumin $(\geq 3.5 \mathrm{~g} / \mathrm{L})$ group according to previously published studies $(10,12)$.

\section{Statistics}

Pre-, peri-, and postoperative covariates are described using mean values \pm standard deviation $(\mathrm{SD})$, median $\left[25^{\text {th }}-75^{\text {th }}\right.$ percentile], or frequencies and percentages. Differences between two groups were evaluated using chi-square test or Fisher's exact tests for categorical variables and unpaired t-tests or Mann-Whitney U tests for continuous variables, depending on whether the assumption of normality holds or not. Univariable and multivariable logistic regression models were fitted to estimate the unadjusted and adjusted effect of low $v s$. normal serum albumin on clinically relevant endpoints, i.e., on AKI, infection, ventilation and transfusion of packed red blood cells. Covariates for the multivariable analyses were identified by the clinical context, without further model selection. Due to the low number of events we did not fit regression models for mortality endpoints. For logistic regression models, odds ratios (OR), corresponding $95 \%$ confidence intervals $(\mathrm{CIs})$, and $\mathrm{P}$ values (Wald test) are given. All reported $\mathrm{P}$ values are two-sided and $\mathrm{P}$ values of $<0.05$ are considered statistically significant. As these are explorative analyses, 
we did not adjust for multiple testing. Statistical analyses were performed using IBM SPSS Statistics version 24 (IBM Corp, Armonk, New York, United States) in cooperation with the Institute of Medical Statistics and Computational Biology at the University of Cologne.

\section{Results}

\section{Baseline parameters}

Preoperative serum albumin levels were completed for 457 of 464 patients (98\%) in this retrospective survey. Patient records were split into low serum albumin [51 patients $(11 \%)$ ] and normal serum albumin [406 patients (89\%)] levels (see Table 1). Patients with low serum albumin levels had significantly more preoperative comorbidities compared to patients with normal serum albumin levels [diabetes ( $45 \%$ versus $30 \%, \mathrm{P}=0.038$ ), dialysis $(12 \%$ versus $3 \%$, $\mathrm{P}=0.009)$, chronic obstructive pulmonary disease (COPD) ( $39 \%$ versus $25 \%, \mathrm{P}=0.042$ ), prior or acute infectious disease $(16 \%$ versus $4 \%, \mathrm{P}=0.003)]$ and a lower ejection fraction below $45 \%$ (39\% versus $22 \%, \mathrm{P}=0.010$ ). Further, patients with preoperative hypoalbuminemia presented lower levels of hemoglobin suggesting anemia $(11 \pm 2$ versus $12 \pm 2 \mathrm{~g} / \mathrm{dL}, \mathrm{P}<0.001)$ as well as elevated creatine levels suggestive of impaired renal function [1.3 (1.0-1.8) versus $1.1(0.9-1.5) \mathrm{mg} / \mathrm{dL}, \mathrm{P}=0.010]$. Risk of perioperative mortality was estimated using log Euroscore and STS risk score systems. Log Euroscore showed no significant differences between groups while the STS risk score predicted a higher risk of mortality for patients with pre-procedural hypoalbuminemia. Other clinical baseline characteristics such as age, body mass index (BMI), atrial fibrillation, prior heart surgery or intervention and transfemoral or non-transfemoral approach did not differ significantly. All patients had severe symptomatic aortic stenosis.

\section{Perioperative complications}

Perioperative and post interventional complications were registered and listed according to the Valve Academic Research Consortium-2 consensus [VARC-2 (15)] shown in Table 2. Patients with hypoalbuminemia needed significantly higher rates of blood and fresh frozen plasma (FFP) transfusions (packed red blood cells: $63 \%$ versus $33 \%$, $\mathrm{P}<0.001$; FFP: $18 \%$ versus $4 \%, \mathrm{P}<0.001$ ). AKI occurred significantly more often in patients with preoperative low serum albumin levels ( $41 \%$ versus $19 \%, \mathrm{P}=0.001)$. Infection and post interventional ventilation, if needed, were administered more often in patients with hypoalbuminemia ( $53 \%$ versus $24 \%$ and $51 \%$ versus $25 \%, \mathrm{P}<0.001)$. Other events like vascular complication, bleeding, stroke, pacemaker implantation, re-thoracotomy, myocardial infarction or tracheotomy were not significantly different between groups.

\section{Outcome}

Patients with preoperative hypoalbuminemia stayed longer in hospital than patients with normal serum albumin levels. The median was 12 and 9 respectively $(\mathrm{P}<0.001)$. Similarly, the length of days in the intensive care unit (ICU) was on average 1 day longer. Five patients (1\%) with preoperative normal serum albumin levels died immediately after TAVR procedure $(<72$ hours) and none in the patient group with pre-procedural low serum albumin levels. We did not find a statistically significant difference for in-hospital mortality ( $8 \%$ versus $3 \%, \mathrm{P}=0.093)$. However, five patients $(10 \%)$ with low and 14 patients (3\%) with normal serum albumin levels died within 30 days $(\mathrm{P}=0.045)$ as shown in Table 3.

\section{Univariable and multivariable regression analysis}

For the endpoint AKI (Table 4), the univariable model revealed a statistically significant difference between albumin groups (low vs. normal albumin: OR, 2.97, 95\% CI, 1.6-5.5, $\mathrm{P}=0.001)$. The magnitude of effect size remained stable in the multivariable model (OR, 3.09, 95\% CI, $1.5-6.5, \mathrm{P}=0.003)$, adjusted for age, gender, diabetes mellitus, hypertension, kidney failure and left ventricular ejection fraction (LVEF) $<45 \%$. The variables were selected according to clinical criteria and all variables considered relevant for adjustment were taken into account. Further, univariable models present a statistically significant higher chance for infection, ventilation und transfusion of packed red blood cells in the low albumin group which also persists in the multivariable regression analysis as shown in Table 4.

\section{Discussion}

Patients with preoperative hypoalbuminemia are commonly patients suffering from severe comorbidities. The baseline characteristics show that patients with low serum albumin levels had (I) significantly lower hemoglobin levels and impaired liver synthesis; (II) reduced cholinesterase activity; (III) significantly more infectious diseases; (IV) impaired 
Table 1 Baseline characteristics and approach according to serum albumin groups

\begin{tabular}{|c|c|c|c|}
\hline Variable & Low albumin $(n=51)$ & Normal albumin $(n=406)$ & $P$ value \\
\hline Female & 18 [35] & $209[51]$ & 0.037 \\
\hline Body mass index $\left(\mathrm{kg} / \mathrm{m}^{2}\right)$ & $26 \pm 5$ & $27 \pm 5$ & 0.328 \\
\hline Hypertension & $46[90]$ & $377[93]$ & $0.773^{*}$ \\
\hline Kidney failure & $24[47]$ & $156[38]$ & 0.287 \\
\hline Dialysis & 6 [12] & $12[3]$ & $0.009^{\star}$ \\
\hline Prior cerebrovascular disease & $11[22]$ & $67[17]$ & 0.215 \\
\hline Chronic obstructive pulmonary disease & 20 [39] & $101[25]$ & 0.042 \\
\hline Pacemaker & $8[16]$ & $42[10]$ & 0.339 \\
\hline Prior coronary bypass & $7[14]$ & $69[17]$ & 0.691 \\
\hline Prior valve implantation & $1[2]$ & $25[6]$ & $0.340^{*}$ \\
\hline New York Heart Association III/IV & $43[84]$ & $259[64]$ & 0.010 \\
\hline Prior malignancy & $14[27]$ & $101[25]$ & 0.733 \\
\hline Prior or acute infectious disease & $8[16]$ & $17[4]$ & $0.003^{*}$ \\
\hline Oral anticoagulation or antiplatelet therapy & $47[92]$ & $359[88]$ & 0.633 \\
\hline Hemoglobin (g/dL) & $11 \pm 2$ & $12 \pm 2$ & $<0.001$ \\
\hline GPT (U/L) & $18(10 ; 33)$ & $18(14 ; 26)$ & 0.340 \\
\hline STS risk score & $6(3 ; 10)$ & $4(3 ; 6)$ & 0.006 \\
\hline Log Euroscore & $12(9 ; 21)$ & $12(8 ; 21)$ & 0.648 \\
\hline \multicolumn{4}{|l|}{ Echocardiographic } \\
\hline Ejection fraction $<45 \%$ & $20[39]$ & $90[22]$ & 0.010 \\
\hline Velocity max (m/s) & $4.1 \pm 1.0$ & $4.2 \pm 1.0$ & 0.364 \\
\hline Mean gradient $(\mathrm{mmHg})$ & $39(30 ; 47)$ & $43(36 ; 52)$ & 0.012 \\
\hline Approach & & & 0.089 \\
\hline Transfemoral approach & 37 [73] & $335[83]$ & \\
\hline Transapical approach & 11 [22] & 60 [15] & \\
\hline Transaortal approach & $3[6]$ & $11[3]$ & \\
\hline
\end{tabular}

Data are presented as number [percentage] for categorical variables, mean standard deviation for normally distributed or median and $\left(25^{\text {th }}\right.$ and $75^{\text {th }}$ percentile) for not normally distributed continuous variables. Accordingly, Chi-square, exact Fisher ${ }^{\star}$, Student's $t$-test or Mann-Whitney U test were used to compare. 
Table 2 Perioperative complication according to serum albumin groups

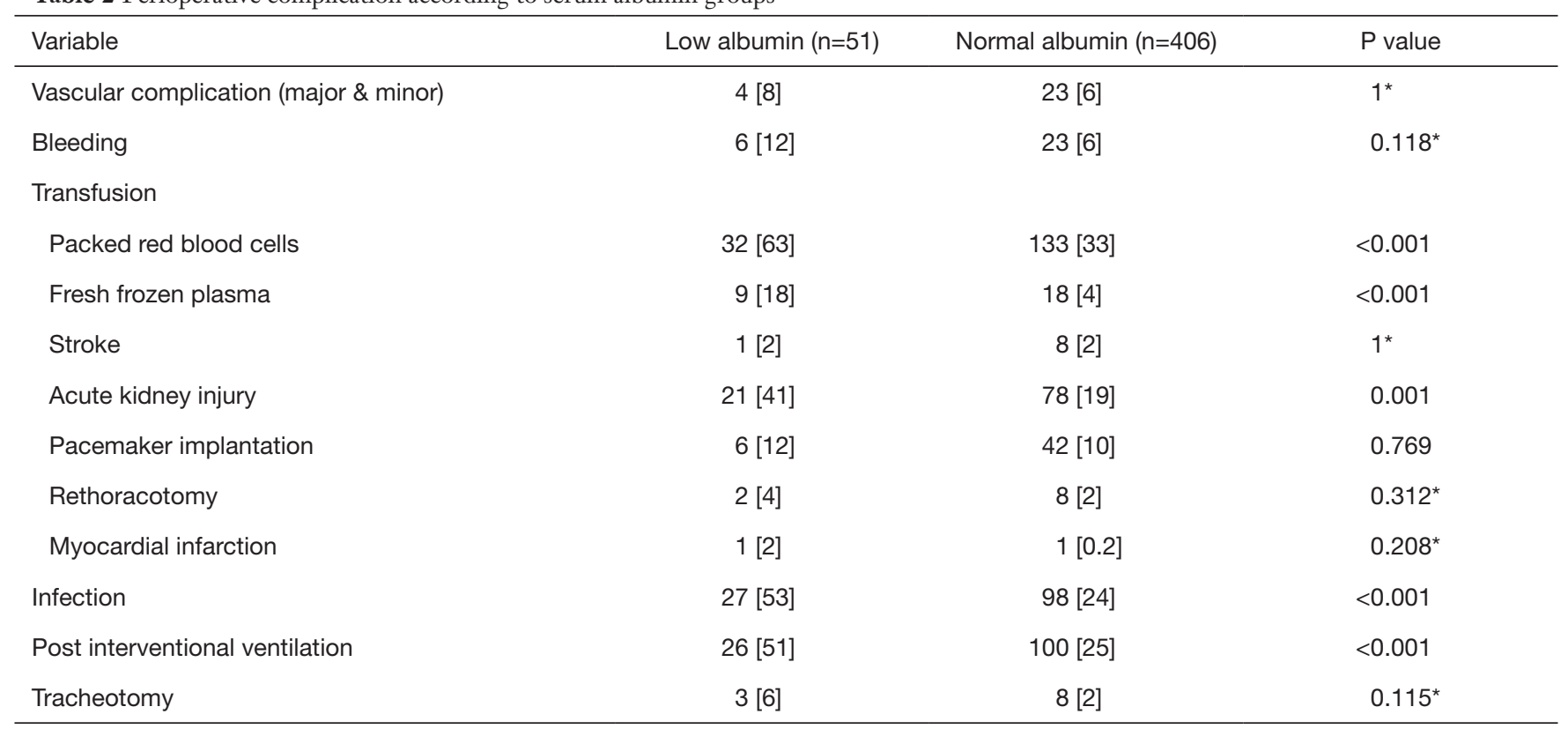

Data presented as number [percentage] for categorical variables, mean standard deviation for normally distributed or median and $\left(25^{\text {th }}\right.$ and $75^{\text {th }}$ percentile) for not normally distributed continuous variables. Accordingly, Chi-square, exact Fisher*, Student's $t$-test or Mann-Whitney $U$ test were used to compare.

Table 3 Hospital stay and mortality rates according to serum albumin groups

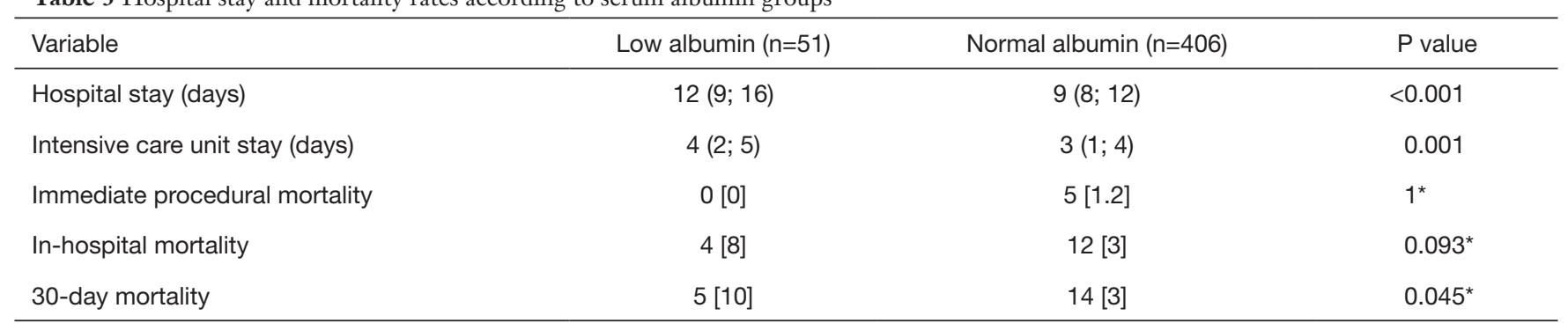

Data presented as number [percentage] for categorical variables, mean standard deviation for normally distributed or median and $\left(25^{\text {th }}\right.$ and $75^{\text {th }}$ percentile) for not normally distributed continuous variables. Accordingly, Chi-square, exact Fisher* ${ }^{\star}$ Student's $t$-test or Mann-Whitney U test were used to compare.

kidney function and (V) signs of prior or acute infectious diseases with antibiotic therapy in advance of surgery.

Some of these can potentially be self-contained. Pathophysiological mechanisms can explain that low serum albumin levels can lead to a decreased colloid-osmotic pressure in plasma and consequently to a lower intravasal volume resulting in a pre-renal acute kidney failure (16). At the same time, patients receive nephrotoxic contrast agents as part of the TAVR procedure which supports periinterventional AKI (17). Need for more transfusions of packed red blood cells and FFP could be explained by the fact that hemoglobin levels were also lower before surgery and that patients suffered from anemia of different causes.

Vascular complications were not significantly different in both groups. Nevertheless, a different surgical technique in the transfemoral approach could avoid access related complications. In most cases, a percutaneous approach is performed for the transfemoral TAVR procedure. Cardounel et al. demonstrated that the use of conscious sedation and surgical cut down for femoral arterial access resulted in a major vascular complication rate of less than $1 \%$ and low in-hospital mortality rates without any significant increase 
Table 4 Uni- and multivariable logistic regression analysis

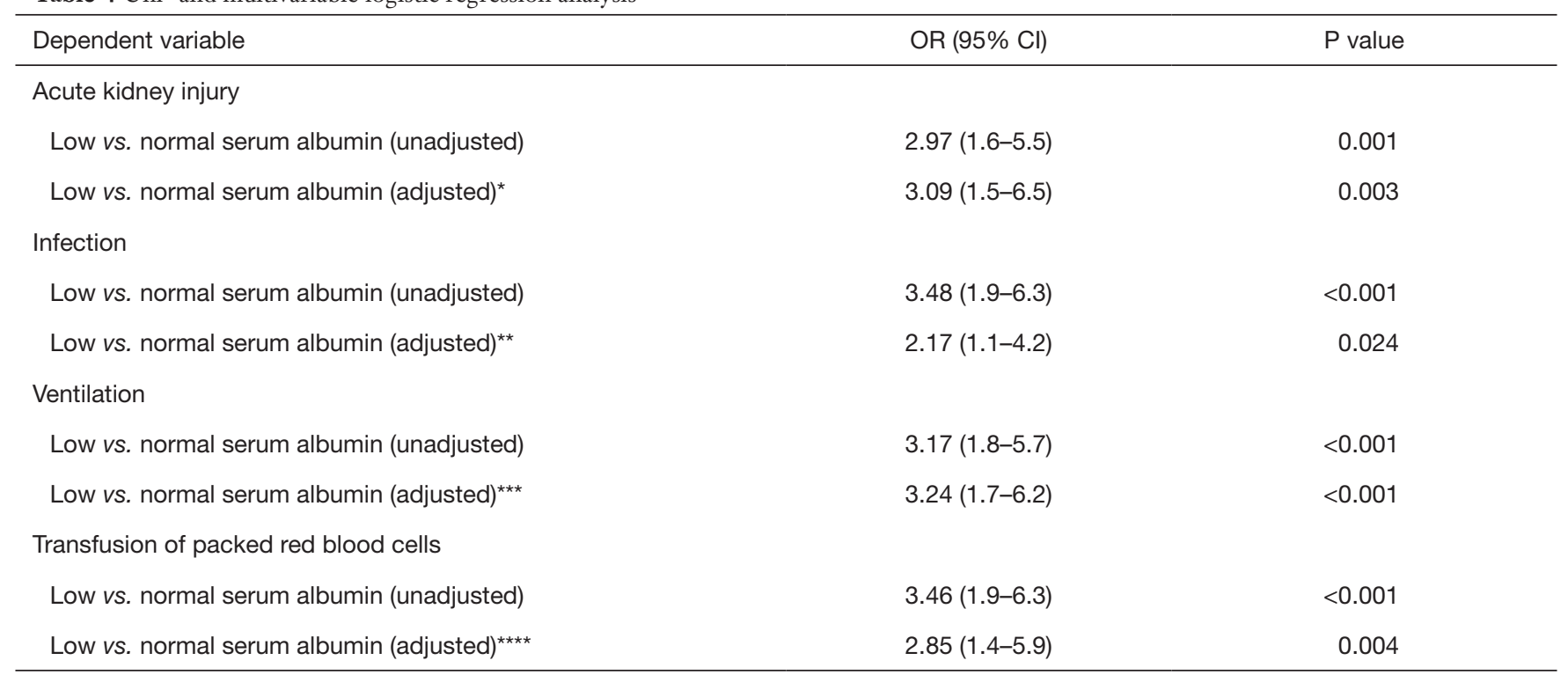

*, adjusted for: age, gender, diabetes mellitus, hypertension, kidney failure, left ventricular ejection fraction $<45 \%$; ${ }^{* *}$, adjusted for: age, gender, diabetes mellitus, chronic obstructive pulmonary disease, prior or acute infectious disease, CRP >20 mg/L; ***, adjusted for: age, gender, chronic obstructive pulmonary disease, coronary artery disease, prior or acute infectious disease, left ventricular ejection fraction $<45 \%$; ${ }^{\star \star \star}$, adjusted for: age, gender, dialyses, prior coronary bypass, prior valve implantation, pre-procedural hemoglobin $\leq 10 \mathrm{~g} / \mathrm{dL}$. OR, odds ratio; CRP, C-reactive protein.

in wound complications (18).

Coronary obstruction is a severe and lethal complication which occurs in less than $1 \%$ of patients during the TAVR procedure. As described by Sultan et al. (19) coronary obstruction was not observed in this cohort.

However, it is clear that patients with hypoalbuminemia show significantly more comorbidities than patients with normal serum albumin levels. This is in stark contrast with a similar study carried out in the MedStar Washington Hospital Center by Koifman et al. (20) who report no significant comorbidities between the low and normal serum albumin groups. Koifman et al. report on equal numbers of patients in the low and normal albumin groups (238 each), while the patient set presented here includes a comparatively small number of patients with hypoalbuminemia $(n=51)$ compared to normal serum albumin levels $(n=406)$.

Importantly, after a 30-day follow-up, more patients with hypoalbuminemia died in this study (10\% as opposed to $3 \%$ ) as well as in the Koifman et al. study [12\% as opposed to 6\% (20)]. The Koifman et al. study expands this to 1 year follow up mortality rates of nearly $30 \%$ in hypoalbuminemic patients compared to $20 \%$ in controls (20). It follows that albumin levels appear to be of prognostic value for patient risk and should be included to risk estimates prior to TAVR procedure.

Further, a trial of pre-procedural albumin substitution in TAVR patients presenting hypoalbuminemia would be timely. In a recent study of coronary bypass surgery, Lee et al. substituted albumin before off pump bypass surgery and compared it with placebo (21). There was no difference in mortality rate among groups, however the authors saw an effect in postoperative renal function. Preprocedural substitution of albumin led to a decreased rate of AKI (21). This study as well as the Koifman et al. study did show differences in mortality rates after TAVR. It is tempting to speculate that albumin substitution could remedy the difference we see between patients with low and normal pre-operative serum albumin levels undergoing TAVR.

The value of this study is limited by its retrospective design, a small group of preprocedural low serum albumin patients and being performed on a single center registry. Furthermore, 30-day mortality cannot be sufficiently evaluated in a uni- and multivariable logistic regression analysis which could support the prognostic value of serum 
albumin and quantify the impact of covariates.

\section{Conclusions}

Patients with hypoalbuminemia reveal severe comorbidities. Mostly, elderly patients undergo TAVR and should be well prepared before the procedure. Our data enforces that serum albumin is an important preoperative marker. Low serum albumin is one of many other preoperative laboratory markers.

\section{Acknowledgements}

None.

\section{Footnote}

Conflicts of Interest: The authors have no conflicts of interest to declare.

Ethical Statement: The study was approved by the Institutional Review Board at the University of Cologne Institutional Review Board. The IRB waived the consent.

\section{References}

1. Chakos A, Wilson-Smith A, Arora S, et al. Long term outcomes of transcatheter aortic valve implantation (TAVI): a systematic review of 5 -year survival and beyond. Ann Cardiothorac Surg 2017;6:432-43.

2. Carnero-Alcázar M, Maroto LC, Cobiella-Carnicer J, et al. Transcatheter versus surgical aortic valve replacement in moderate and high-risk patients: a meta-analysis. Eur J Cardiothorac Surg 2017;51:644-52.

3. Mack MJ, Leon MB, Smith CR, et al. 5-year outcomes of transcatheter aortic valve replacement or surgical aortic valve replacement for high surgical risk patients with aortic stenosis (PARTNER 1): a randomised controlled trial. Lancet 2015;385:2477-84.

4. Schofer J. Who comes off best with closed chest? Aortic valve replacement in patients with high surgical risk. J Am Coll Cardiol 2014;63:1110-1.

5. Kapadia SR, Leon MB, Makkar RR, et al. 5-year outcomes of transcatheter aortic valve replacement compared with standard treatment for patients with inoperable aortic stenosis (PARTNER 1): a randomised controlled trial. Lancet 2015;385:2485-91.

6. Leon MB, Smith CR, Mack M, et al. Transcatheter aortic- valve implantation for aortic stenosis in patients who cannot undergo surgery. N Engl J Med 2010;363:1597-607.

7. Brennan JM, Thomas L, Cohen DJ, et al. Transcatheter Versus Surgical Aortic Valve Replacement: PropensityMatched Comparison. J Am Coll Cardiol 2017;70:439-50.

8. Nashef SA, Roques F, Michel P, et al. European system for cardiac operative risk evaluation (EuroSCORE). Eur J Cardiothorac Surg 1999;16:9-13.

9. Vassileva CM, Aranki S, Brennan JM, et al. Evaluation of The Society of Thoracic Surgeons Online Risk Calculator for Assessment of Risk in Patients Presenting for Aortic Valve Replacement After Prior Coronary Artery Bypass Graft: An Analysis Using the STS Adult Cardiac Surgery Database. Ann Thorac Surg 2015;100:2109-15; discussion 2115-6.

10. Gatta A, Verardo A, Bolognesi M. Hypoalbuminemia. Intern Emerg Med 2012;7 Suppl 3:S193-9.

11. Wiedermann CJ, Wiedermann W, Joannidis M. Causal relationship between hypoalbuminemia and acute kidney injury. World J Nephrol 2017;6:176-87.

12. Karas PL, Goh SL, Dhital K. Is low serum albumin associated with postoperative complications in patients undergoing cardiac surgery? Interact Cardiovasc Thorac Surg 2015;21:777-86.

13. Vincent JL, Dubois MJ, Navickis RJ, et al. Hypoalbuminemia in acute illness: is there a rationale for intervention? A meta-analysis of cohort studies and controlled trials. Ann Surg 2003;237:319-34.

14. Keller F, Feldmann K, Metz FJ. Eine verbesserte Methode zur selektiven Albuminbestimmung mit Bromkresolgrün auf dem SMAR 12/60. 1981 J Clin Chem Gin Biochem 1981;19:169-71.

15. Kappetein AP, Head SJ, Genereux P, et al. Updated standardized endpoint definitions for transcatheter aortic valve implantation: the Valve Academic Research Consortium-2 consensus document (VARC-2). Eur J Cardiothorac Surg 2012;42:S45-60.

16. Lee EH, Baek SH, Chin JH, et al. Preoperative hypoalbuminemia is a major risk factor for acute kidney injury following off-pump coronary artery bypass surgery. Intensive Care Med 2012;38:1478-86.

17. Scherner $M$, Wahlers T. Acute kidney injury after transcatheter aortic valve implantation. J Thorac Dis 2015;7:1527-35.

18. Cardounel A, Gleason TG, Lee JS, et al. Surgical cut down for vascular access with conscious sedation for transcatheter aortic valve replacement: the best of both worlds? Interact Cardiovasc Thorac Surg 2018;27:494-7.

19. Sultan I, Siki M, Wallen T et al, Management of 
coronary obstruction following transcatheteraortic valve replacement. J Card Surg 2017;32:777-81.

20. Koifman E, Magalhaes MA, Ben-Dor I, et al. Impact of pre-procedural serum albumin levels on outcome of patients undergoing transcatheter aortic valve replacement. Am J Cardiol 2015;115:1260-4.

Cite this article as: Gassa A, Borghardt JH, Maier J, Kuhr K, Michel M, Ney S, Eghbalzadeh K, Sabashnikov A, Rudolph T, Baldus S, Mader N, Wahlers T. Effect of preoperative low serum albumin on postoperative complications and early mortality in patients undergoing transcatheter aortic valve replacement. J Thorac Dis 2018;10(12):6763-6770. doi: 10.21037/jtd.2018.11.30
21. Lee EH, Kim WJ, Kim JY, et al. Effect of Exogenous Albumin on the Incidence of Postoperative Acute Kidney Injury in Patients Undergoing Off-pump Coronary Artery Bypass Surgery with a Preoperative Albumin Level of Less Than 4.0 g/dL. Anesthesiology 2016;124:1001-11. 